\title{
Recent results in hyperon physics from the NA48 and KTeV experiments
}

\author{
C. Lazzeroni ${ }^{\mathrm{a} *}$ \\ ${ }^{a}$ Cavendish Laboratory, University of Cambridge, \\ JJ Thomson Avenue, Cambridge CB3 0HE, United Kingdom
}

Recent results from the NA48 and KTeV experiments on neutral hyperon decays are presented.

\section{INTRODUCTION}

The NA48 experiment was performed at the CERN SPS accelerator and used a $400 \mathrm{GeV} / \mathrm{c}$ proton beam impinging on a Be target to produce a neutral beam [1]. In 2002, the beam line was modified to increase the $K_{S}$ and neutral hyperon intensity while the $K_{L}$ line was blocked. The target position and the production angle $(-4.2 \mathrm{mrad})$ were chosen in such a way that the beam axis passed through the center of the electromagnetic calorimeter [2]. In order to minimize the interaction of the neutral beam with air, the final collimator was followed by a $90 \mathrm{~m}$ long evacuated tank terminated by a $0.3 \% X_{0}$ thick kevlar window. The NA48 detector [1] was located downstream of this region in order to collect the products of the particles decaying in the volume contained by the tank. For a detailed description of the detector and the trigger, see [2]. On average, about $1.4 \times 10^{4} \Xi^{0}$ per spill, with energy between 70 and $220 \mathrm{GeV}$, decayed in the fiducial volume (vertex position bewteen $5 \mathrm{~m}$ and $50 \mathrm{~m}$ from the target). In this fiducial volume large numbers of $\Xi^{0} \rightarrow \Lambda \pi^{0}$ and $\overline{\Xi^{0}} \rightarrow \bar{\Lambda} \pi^{0}$ were recorded: $N\left(\Xi^{0}\right)=\left(2.422 \pm 0.003_{\text {stat }} \pm 0.018_{\text {syst }}\right) \cdot 10^{9}$, $N\left(\overline{\Xi^{0}}\right)=\left(2.254 \pm 0.012_{\text {stat }} \pm 0.017_{\text {syst }}\right) \cdot 10^{8}$ and mainly used for normalizating the various branching ratio measurements. The accumulated statistics allow high precision studies of $K_{S}$ and hyperons.

The $\mathrm{KTeV}$ experiment took place at the Tetravon accelerator. The data analysed here were taken in 1999 during the E799-II configuration of $\mathrm{KTeV}$ [3]. An intense $800 \mathrm{GeV} / \mathrm{c}$ proton

\footnotetext{
*On behalf of the NA48 and $\mathrm{KTeV}$ Collaborations
}

beam was directed onto a $\mathrm{BeO}$ target. After a system of collimators, converters and dipoles, the surviving neutral beams entered a $65 \mathrm{~m}$ long vacuum tank, that defined the decay region, which began $94 \mathrm{~m}$ from the target. The $\mathrm{KTeV}$ detector was located downstream of this region; for a detailed description of the detector and the trigger, see [3]. About $3 \times 10^{8} \Xi^{0}$ decayed in the decay region, with momentum peaked at $290 \mathrm{GeV} / \mathrm{c}$. The sweeping magnets in the beamline were designed and operated so that the integrated magnetic field delivered $\Xi^{0}$ hyperons polarised (with about $10 \%$ polarization) in the positive or negative vertical direction. The magnetic field was reversed regularly so that the net polarization was zero for the data discussed here.

\section{2. $\Xi^{0}$ BETA DECAYS}

The study of hadron beta decays gives important information on the interplay between the weak interaction and hadron structure, determined by the strong interaction. In this context the $\Xi^{0}$ beta decay allows testing both of $\mathrm{SU}(3)$ symmetry, via its strong analogy with the wellknown neutron beta decay, and of the quark mixing model, via extraction of $V_{u s}$ (the sine of the Cabibbo angle).

NA48 collected a sample of $6316 \Xi^{0} \rightarrow \Sigma^{+} e^{-} \bar{\nu}_{e}$ events (with the $\Sigma^{+}$decaying into $p \pi^{0}$ ) with energy between 70 and $220 \mathrm{GeV}$ and decay vertex between $5 \mathrm{~m}$ and $50 \mathrm{~m}$ from the final collimator. With a background of about $2 \%$, a value for the branching ratio has been extracted: $B R\left(\Xi^{0} \rightarrow\right.$ $\left.\Sigma^{+} e^{-} \bar{\nu}_{e}\right)=\left(2.51 \pm 0.03_{\text {stat }} \pm 0.09_{\text {syst }}\right) 10^{-4}$, where the systematic error is dominated by the trigger efficiency determination, the geometrical ac- 
ceptance and the form factors. This value is in agreement with the $\mathrm{KTeV}$ measurement, based on 625 events: $B R_{K T e V}\left(\Xi^{0} \rightarrow \Sigma^{+} e^{-} \bar{\nu}_{e}\right)=$ $\left(2.54 \pm 0.11_{\text {stat }} \pm 0.16_{\text {syst }}\right) 10^{-4}[4]$. Including the dependence of the form factors on the momentum transfer and the radiative corrections, the value for $V_{u s}$ extracted from the branching ratio measurement is: $V_{u s}=0.208 \pm 0.006{ }_{-0.025}^{+0.030} g_{1} / f_{1}$, in agreement with the Standard Model expection of $0.2274 \pm 0.0021[5]$. The systematic error is largely dominated by the error on the ratio of the form factors $g_{1} / f_{1}=1.267 \pm 0.035$ taken from PDG. Alternatively, a value for $g_{1} / f_{1}$ can be extracted from the measured branching ratio, using $V_{u s}=$ 0.2257 [5], giving: $g_{1} / f_{1}=1.20 \pm 0.04_{b r} \pm 0.03_{\text {ext }}$, where the last uncertainty includes contributions from $V_{u s}, \Xi^{0}$ lifetime and form factor $f_{2} / f_{1}$. The agreement with the prediction for exact $\mathrm{SU}(3)$ symmetry favours $\mathrm{SU}(3)$ breaking models that leave $g_{1} / f_{1}$ unchanged.

A sample of $102 \Xi^{0} \rightarrow \Sigma^{+} \mu^{-} \overline{\nu_{\mu}}$ events was selected, with a background of $32 \pm 3.0$ (see Fig. 1). Using the electron decay as normalization channel, the measurement of the muon branching ratio was extracted: $B R\left(\Xi^{0} \rightarrow \Sigma^{+} \mu^{-} \bar{\nu}_{\mu}\right)=$ $\left(2.2 \pm 0.3_{\text {stat }} \pm 0.2_{\text {syst }}\right) 10^{-6}$. This is the largest sample of semi-muonic decays so far collected.

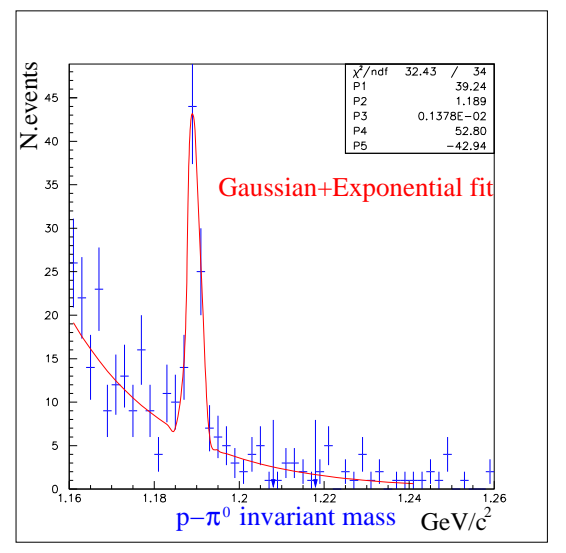

Figure 1. Distribution of $p \pi^{0}$ invariant mass for selected events with negative muon in NA48. A clear signal for $\Sigma^{+}$is visible.
$\mathrm{KTeV}$ collected a sample of $9 \Xi^{0} \rightarrow \Sigma^{+} \mu^{-} \bar{\nu}_{\mu}$ events (with the $\Sigma^{+}$decaying into $p \pi^{0}$ ), and a sample of $1139 \Xi^{0} \rightarrow \Sigma^{+} e^{-} \bar{\nu}_{e}$ for normalization purpose, requiring 2 charged particles (one being an electron or a muon) and 2 neutral clusters. The distribution of events that survived the selection is shown in a plot of $p_{t}^{2}$ (the square of the transverse momentum relatve to the beam) of the $\Sigma^{+} \mu^{-}$versus the invariant mass of the $p \pi^{0}$ system (see Fig. 2). For details on the selection, see [3]. The box is defined by the Monte Carlo simulation to accept $90 \%$ of the signal events. There are 9 signal events inside the box, clustered around $1.189 \mathrm{GeV} / \mathrm{c}^{2}$, and 1 event outside. The background, evaluated using Monte Carlo simulation and data with wrong sign assignements for tracks, was found to be negligible inside the signal box; 54 background events were subtracted from the normalization channel. Using these samples, the ratio of the decay rates is measured to be $\Gamma\left(\Xi^{0} \rightarrow\right.$ $\left.\Sigma^{+} \mu^{-} \bar{\nu}_{\mu}\right) / \Gamma\left(\Xi^{0} \rightarrow \Sigma^{+} e^{-} \bar{\nu}_{e}\right)=\left(1.8_{-0.5}^{+0.7}(\right.$ stat $) \pm$ $0.2($ syst $)) \times 10^{-2}$. The systematic uncertainties receive contributions from the statistics in the normalization mode, the ratio of acceptances, and the muon identification efficiency. Using the value $\operatorname{Br}\left(\Xi^{0} \rightarrow \Sigma^{+} e^{-} \bar{\nu}_{e}\right)=(2.7 \pm 0.4) \times 10^{-4}$ [6], the branching ratio for the muon semileptonic decay is found to be: $\operatorname{Br}\left(\Xi^{0} \rightarrow \Sigma^{+} \mu^{-} \bar{\nu}_{\mu}\right)=$ $\left(4.7_{-1.4}^{+2.0}(\right.$ stat $) \pm 0.8($ syst $\left.)\right) \times 10^{-6}$, where the systematic error also includes the contribution from the uncertainty of the electron beta decay.

\section{ASYMMETRY IN RADIATIVE DE- CAYS}

The weak radiative hyperon decays are quite accessible experimentaly, in terms of the branching ratio and the asymmetry of the baryon emission with respect to the initial spin. However, despite their simplicity, there are theoretical difficulties in explaining these decays, mainly because predictions using the quark model do not match predictions from an analysis at the hadron level[7]. Hara theorem proved that the asymmetry is zero in the $\mathrm{SU}(3)$ limit for $\Xi^{-}$and $\Sigma^{+}$decays, assuming $\mathrm{CP}$ invariance and U-spin symmetry[10]. An estimate, based on single quark $s \rightarrow d$ transitions and that takes into account 


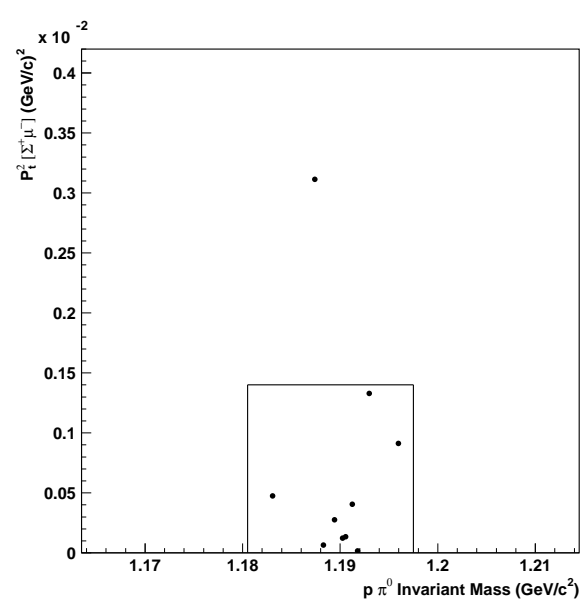

Figure 2. Distribution of $p \pi^{0}$ invariant mass for selected events with negative muon in $\mathrm{KTeV}$.

SU(3) breaking, predicts a modest positive asymmetry[11]. However, the only asymmetry been accurately measured so far is $-0.76 \pm 0.08$ for $\Sigma^{+} \rightarrow p \gamma[12]$. To constrain the theoretical models, it is important to measure accurately the parameters of weak radiative decays for all hyperons.

In the decay $\Xi^{0} \rightarrow \Lambda \pi^{0}$, the proton angular distribution with respect to the $\Xi^{0}$ direction in the $\Lambda$ rest frame can be written as $d N / d \cos \Theta_{\Lambda}=N_{0}\left(1+\alpha_{\Xi} \alpha_{\Lambda} \cos \Theta_{\Lambda}\right)$, where $\alpha_{\Xi}$ is the weak decay asymmetry and $\alpha_{\Lambda}=0.642$ is the $\Lambda$ weak decay asymmetry. A similar formula is valid for $\Xi^{0} \rightarrow \Lambda \gamma$, where the sign minus can be understood in terms of fundamental angular momentum considerations [5]: $d N / d \cos \Theta_{\Lambda}=$ $N_{0}\left(1-\alpha_{\Xi} \alpha_{\Lambda} \cos \Theta_{\Lambda}\right)$. This can be extended to the $\Xi^{0} \rightarrow \Sigma^{0} \gamma$ decay, considering the angles $\Theta_{\Xi \Lambda}$ of the $\Lambda$ direction with respect to the $\Xi$ direction in the $\Sigma$ rest frame, and $\Theta_{\Sigma p}$ of the proton direction with respect to the $\Sigma$ direction in the $\Lambda$ rest frame: $d^{2} N / d \cos \Theta_{\Xi \Lambda} d \cos \Theta_{\Sigma p}=$ $N_{0}\left(1+\alpha_{\Xi} \alpha_{\Lambda} \cos \Theta_{\Xi \Lambda} \cos \Theta_{\Sigma p}\right)=N_{0}\left(1+\alpha_{\Xi} \alpha_{\Lambda} x\right.$.

From the 2002 data, NA48 selected $\sim 4 \times 10^{6}$ $\Xi^{0} \rightarrow \Lambda \pi^{0}, \sim 52600 \Xi^{0} \rightarrow \Lambda \gamma$ and $\sim 15600$ $\Xi^{0} \rightarrow \Sigma^{0} \gamma$ events. The background is respectively $0.1 \%$ (mainly from $\Xi^{0} \rightarrow \Sigma^{0} \gamma$ ), $0.7 \%$ (mainly from $\Xi^{0} \rightarrow \Lambda \pi^{0}$, fake $\Lambda$ s and fragments of 2 events overlapping in time), and $3 \%$ (mainly from $\Xi^{0} \rightarrow$ $\left.\Lambda \pi^{0}\right)$. From these samples, measurements of the decay asymmetries were obtained from the respective distributions of $\cos \Theta$ (or $x$ ); the obtained values are shown in Table1, together with the previous measurements to which they all agree. In all cases, the NA48 measurements represent a significant improvement in precision. The major sources of systematic uncertainties come from the acceptance correction and the trigger efficiency.

Table 1

Preliminary NA48 measurements of asymmetries for weak $\Xi^{0}$ decays, compared to the previous ones from literature.

\begin{tabular}{ll}
\hline$\alpha_{\Xi} \alpha_{\Lambda}\left(\Lambda \pi^{0}\right)$ & $-0.282 \pm 0.003_{\text {stat }} \pm 0.028_{\text {syst }}$ \\
$\mathrm{PDG}$ & $-0.264 \pm 0.013$ \\
\hline$\alpha_{\Xi} \alpha_{\Lambda}(\Lambda \gamma)$ & $-0.439 \pm 0.013_{\text {stat }} \pm 0.038_{\text {syst }}$ \\
$\mathrm{NA} 48(1999)[8]$ & $-0.50 \pm 0.13$ \\
\hline$\alpha_{\Xi} \alpha_{\Lambda}(\Sigma \gamma)$ & $-0.438 \pm 0.020_{\text {stat }} \pm 0.041_{\text {syst }}$ \\
$\mathrm{KTeV}[9]$ & $-0.40 \pm 0.06$ \\
\hline
\end{tabular}

\section{4. $\Xi^{0}$ LIFETIME}

Several experiments in the 1960s and 1970s performed measurements of the $\Xi^{0}$ lifetime, yielding a world average, based on an integrated data sample of less than 8000 events, of $\Gamma\left(\Xi^{0}\right)=(2.90 \pm$ $0.09) \times 10^{-10} s[5]$. In the last 30 years or so, no new measurements have been published. The $\Xi^{0}$ lifetime is an important input to other $\Xi^{0}$-related parameters, like $V_{u s}$ from hyperon beta decays, and is also theoretically interesting. When assuming the full validity of the $\Delta I=1 / 2$ rule in hadronic weak decays, the decay matrix elements of the neutral and charged $\Xi \rightarrow \Lambda \pi$ should simply be connected by $M\left(\Xi^{0} \rightarrow \Lambda \pi^{0}\right)=M\left(\Xi^{-} \rightarrow\right.$ $\left.\Lambda \pi^{-}\right) / \sqrt{2}$ because of isospin arguments. Taking into account the slightly different masses of the particles involved, as well as the rare decays to other final states[5], the lifetimes should follow the relation $\Gamma\left(\Xi^{0}\right) / \Gamma\left(\Xi^{-}\right) \approx 2.031$. The comparison of measured lifetimes is a good test for $\Delta I=1 / 2$ violating effects. Using the previous 
measured lifetimes [5], the experimental value of the ratio is $1.77 \pm 0.06$.

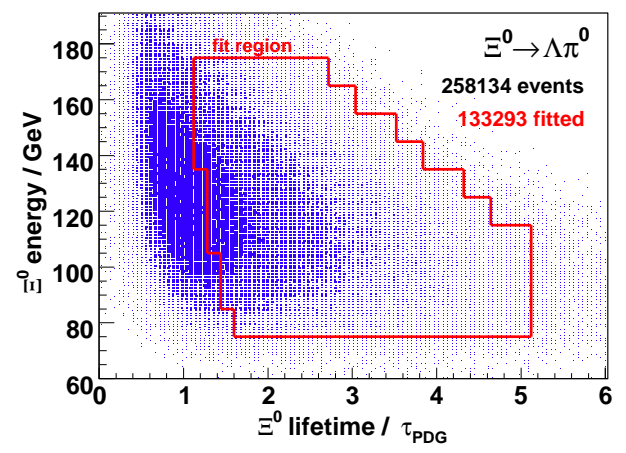

Figure 3. Distribution of $\Xi^{0}$ energy versus $\Xi^{0}$ lifetime in units of PDG lifetime.

From a sample of $\sim 258000 \Xi^{0} \rightarrow \Lambda \pi^{0}$ events collected in NA48 with a minimum bias trigger, the $\Xi^{0}$ lifetime has been measured for decays that occur outside the final collimator. The distribution of $\Xi^{0}$ energy versus $\Xi^{0}$ lifetime in units of PDG lifetime is shown in Fig. 3 for collected events; the contour indicates the events used for the lifetime measurements. Distributions of lifetime for data and Monte Carlo events (simulated with the PDG lifetime) were produced and their ratio was fitted in 10 energy bins of $10 \mathrm{GeV}$ each, from $75 \mathrm{GeV}$ to $175 \mathrm{GeV}$. The distribution of the ratio, summed over all energy bins, is shown in Fig. 4. The distribution of the fit results, expressed as relative deviations from the PDG measurement, is flat in bins of $\Xi^{0}$ energy. The preliminary value obtained is $\tau\left(\Xi^{0}\right) / \tau_{P D G}=$ $1.0626 \pm 0.0044_{\text {stat }} \pm 0.0043_{\text {syst }}$ which corresponds to a lifetime of $\tau\left(\Xi^{0}\right)=\left(3.082 \pm 0.013_{\text {stat }} \pm\right.$ $\left.0.012_{\text {syst }}\right) \times 10^{-10} \mathrm{~s}$. Using the measured value for $\Gamma\left(\Xi^{-}\right)=(1.639 \pm 0.015) \times 10^{-10} s[5]$, the ratio of the neutral over charged lifetime is found to be $1.880 \pm 0.020$.

\section{CONCLUSIONS}

Recent results from the NA48 and $\mathrm{KTeV}$ experiments on neutral hyperon decays have been

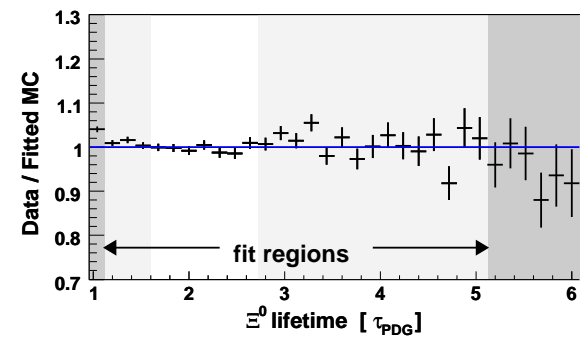

Figure 4. Ratio of lifetimes for data and Monte Carlo events (simulated with the PDG lifetime) as a function of $\Xi^{0}$ lifetime.

presented. Preliminary measurements have been summarised for the $\Xi^{0}$ muon-beta decay for both experiments; preliminary values from NA48 for the $\Xi^{0}$ lifetime and for weak decay asymmetries in various decay channels have been shown.

\section{REFERENCES}

1. J. R. Batley et al., Phys.Lett. B 544 (2002) 97.

2. NA48/1 Status Report SPSC/M670, CERN/SPSC 2001-029 (2001).

3. A. Alavi-Harati, hep-ex/0504055, 2005.

4. A. Alavi-Harati, hep-ex/0105016, 2001.

5. Particle Data Group, Journal of Physics G 33 (2006).

6. Review of Particle Physics, Phys. Lett. B 592 (2004).

7. J. Lach, P. Zenczykowski, Int. Jour. Mod. Phys. A 10 (1995).

P. Zenczykowski, Phys. Rev. D 62 (2000).

P. Zenczykowski, Proceedings of BEACH 2006 Conference, Lancaster, UK (2006).

8. J. R. Batley et al., Phys.Lett. B 584 (2004).

9. A. Alavi-Harati, Phys. Rev. Lett. 86 (2001).

10. Y. Hara, Phys. Rev. Lett. 12 (1964).

11. N. vasanti, Phys. Rev. D 13 (1976).

12. M. Foucher et al., Phys. Rev. Lett. 68 (1992). 